\title{
Secondary Primary Prostate Cancer after Colorectal Cancer: A Nationwide Population-based Cohort Study in Korea
}

ORIGINAL

ARTICLE

\section{Hyun Soo Kim ${ }^{1, *}$, Yoon Jin Choi ${ }^{1, *}$, Dong Woo Shin ${ }^{1}$, Kyung-Do Han ${ }^{2}$, Hyuk Yoon ${ }^{1}$, Cheol Min Shin ${ }^{1}$, Young Soo Park ${ }^{1}$, Nayoung Kim ${ }^{1,3}$, Dong Ho Lee ${ }^{1,3}$}

${ }^{1}$ Department of Internal Medicine, Seoul National University Bundang Hospital, Seongnam, ${ }^{2}$ Department of Biostatistics, College of Medicine, The Catholic University of Korea, ${ }^{3}$ Department of Internal Medicine and Liver Research Institute, Seoul National University College of Medicine, Seoul, Korea

Background: Colorectal cancer (CRC) and prostate cancer frequently occur in developed countries. There are several reports on the association between CRC and prostate cancer; however, the conclusions are inconsistent to investigate the association of the development of secondary primary prostate cancer among patients with prior primary CRC using a nationwide population-based dataset. Methods: Patients registered in the Republic of Korea National Health Insurance System database who were diagnosed with CRC between 2007 and 2012 were followed-up until the end of 2015, and we investigated the new diagnosis secondary primary prostate cancer. We compared the incidence of prostate cancer in age-matched controls using the Cox proportional hazards models.

Results: We analyzed a total of 85,455 first primary CRC survivors. During the follow-up period of 494,222 person-years, 2,005 patients (2.30\%) developed secondary primary prostate cancer (incidence rate 4.06/1,000 person-years). The median duration of follow-up was 5.78 years. Compared with the general population, CRC patients had a significantly increased risk of secondary primary prostate cancer $(\mathrm{HR}=2.30,95 \% \mathrm{Cl}=2.18-2.43 ; P<0.001)$. Multivariate analysis (including age, sex, body mass index, hypertension, diabetes mellitus, dyslipidemia, and income) showed that age $<55$ years $(H R=20.74,95 \% \mathrm{Cl}=11.81-36.41 ; P<0.001)$ is a significant independent predictor of secondary primary prostate cancer development.

Conclusions: Men diagnosed with colorectal cancer are at an increased risk of secondary primary prostate cancer, particularly those aged $<55$ years. The data suggests that colorectal cancer patients aged $<55$ years require regular screening for prostate cancer.

(J Cancer Prev 2017;22:241-247)

Key Words: Colorectal neoplasm, Prostatic neoplasm, Second primary neoplasms

\section{INTRODUCTION}

Cancer is the second leading cause of death worldwide after cardiovascular diseases. Among all cancers, colorectal cancer (CRC) is the third most common malignancy in men and the second most common malignancy in women worldwide. In 2015. 1.7 million incidences of CRC occurred globally, and the resultant 832,000 patients died. ${ }^{1,2}$ Due to the improved survival rates resulting from early diagnosis and improved treatment, the survival of cancer patients has increased, and this trend will continue. ${ }^{3}$ Therefore, late outcomes of CRC survivors resulting in complications, such as increased risk of second primary malignancies (SPMs), has become an important issue. ${ }^{4}$ Prostate cancer, the most common cancer diagnosis, is the third leading cause of death in men, with more than 1.6 million new cases in $2015 .{ }^{1.5}$ Recently, increasing evidence supports the hypothesis that metabolic syndrome is involved in the development and progression of certain types of malignancies; ${ }^{6}$ thus, it can be

Received November 30, 2017, Revised December 15, 2017, Accepted December 15, 2017

Correspondence to: Dong Ho Lee

Department of Internal Medicine, Seoul National University Bundang Hospital, 82 Gumi-ro 173beon-gil, Bundang-gu, Seongnam 13520, Korea

Tel: +82-31-787-7009, Fax: +82-31-787-4051, E-mail: dhljohn@yahoo.co.kr, ORCID: Dong Ho Lee, http://orcid.org/0000-0002-6376-410X

*These authors contributed equally to this work as co-first authors.

Copyright (C) 2017 Korean Society of Cancer Prevention

(c) This is an Open Access article distributed under the terms of the Creative Commons Attribution Non-Commercial License (http://creativecommons.org/licenses/by-nc/4.0) which permits unrestricted non-commercial use, distribution, and reproduction in any medium, provided the original work is properly cited. 
inferred that $\mathrm{CRC}$ may share common risk factors for several metabolic syndrome-related cancers, including prostate cancer. The prevalence of CRC and prostate cancer in Asian countries has increased, and this may be due to the adoption of a westernized lifestyle, and subsequently increasing incidence of metabolic syndrome. ${ }^{7}$

Although some studies have reported the occurrence of secondary primary prostate cancer (SPPC) after CRC, the results are inconsistent. ${ }^{2}$ Furthermore, some clinicians have demonstrated a high incidence of SPPC in CRC patients, but others have not confirmed any such results. ${ }^{8-11}$ Thus, knowledge of the incidence of SPPC in CRC survivors is necessary for an effective surveillance program as well as to direct attention to organs vulnerable to secondary malignancies. ${ }^{12}$ Using this background, we analyzed the cohort data from the National Health Insurance System (NHIS) in Korea, to investigate the association of the development of SPPC among patients with prior primary CRC.

\section{MATERIALS AND METHODS}

\section{Data source}

We analyzed data from the NHIS database, registration for which is mandatory for all Koreans. NHIS is responsible for the national health checkup programs, which include a general health examination for all insured employees or self-employed persons aged $>40$ years. NHIS recommends a semi-compulsive health checkup to be undertaken at least biennially. The NHIS database includes an eligibility database (age, sex, socioeconomic variables, type of eligibility, and income level), a medical treatment database (based on the medical records by International Statistical Classification of Diseases and Related Health Problems (ICD) codes that were claimed by medical service providers for their medical expense claims), a health examination database (results of general health examinations and questionnaires on lifestyle and behavior), and a medical care institution database (types of medical care institutions, location, equipment, and number of physicians). ${ }^{13-15}$ Since the study involved routinely collected data, obtaining informed consent was not required. All procedures involving human participants were performed in accordance with the ethical standards of the institutional and national research committees, and 1964 Helsinki declaration including its later amendments or comparable ethical standards. The study was approved by the Institutional Review Board of Seoul National University Bundang Hospital (X-1708-417-905).

\section{Study population}

The incidences of SPPC among men diagnosed with CRC (ICD-10 codes C18, C19, and C20) were compared with those of randomly selected controls. The control group, from the general population, was matched to CRC patients, according to age at a $1: 5$ ratio. The primary outcome was the diagnosis of SPPC (ICD-10 codes C61) after a diagnosis of CRC. To exclude the possibility of synchronous or metastatic CRC, patients with a diagnosis of a prostate cancer within a latency period of 1 year after CRC diagnosis were excluded. This cohort was followed-up from January 1, 2007 until December 31, 2015, to identify the development SPPC.

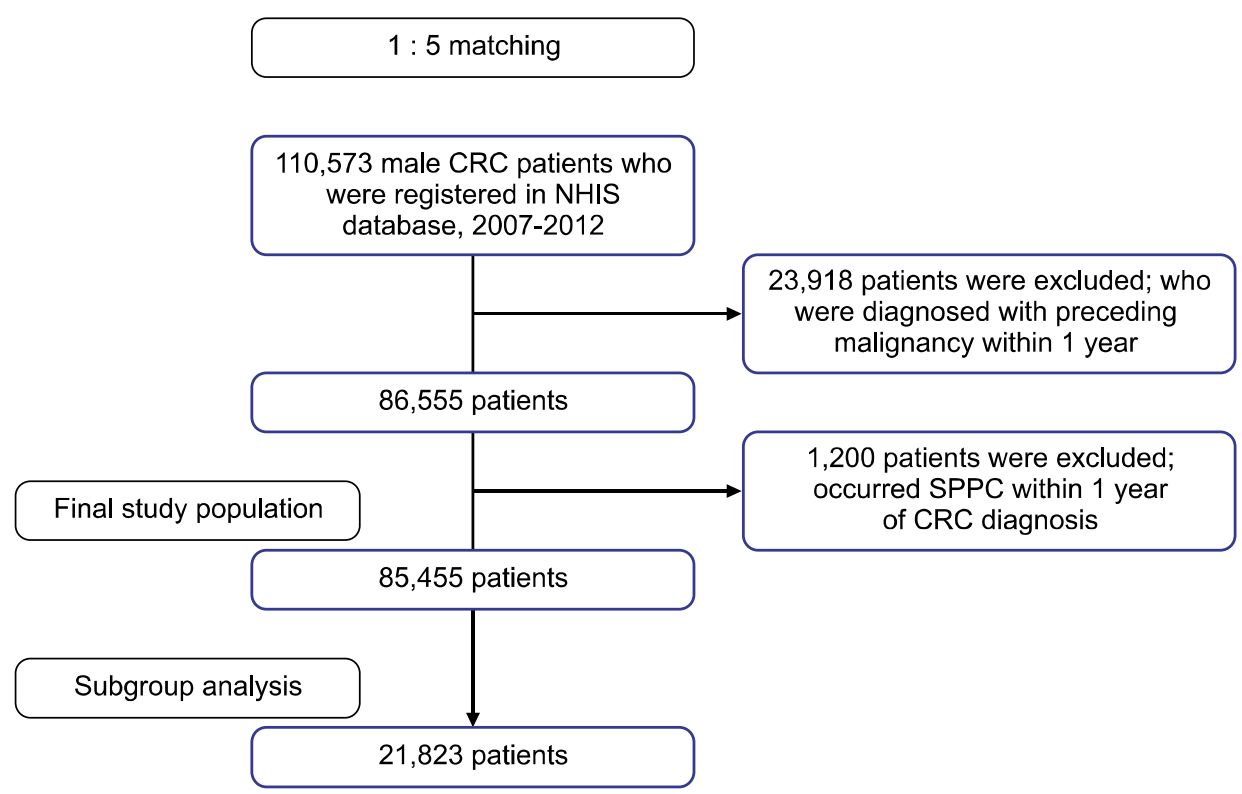

Figure 1. CONSORT flow diagram of patient recruitment. CRC, colorectal cancer; NHIS, National Health Insurance System; SPPC, secondary primary prostate cancer. 
In addition, people previously diagnosed with cancer other than CRC were excluded. Information about the date of diagnosis of previous CRC, latency period, yearly income, and comorbidities (diabetes mellitus, hypertension, and dyslipidemia) was extracted from the database. Finally we enrolled a total of 85,455 CRC patients. We performed additional subgroup analysis for people ( $\mathrm{n}$ = who had health checkups within 1 year before CRC diagnosis). In this analysis, body mass index (BMI), alcohol consumption, exercise, and smoking were included as confounding factors in addition to age, sex, income and residence, diabetes mellitus, hypertension, and dyslipidemia. This is summarized in Figure 1.

\section{Definitions}

Diabetes mellitus was defined as fasting blood glucose $\geq 126 \mathrm{mg} / \mathrm{dL}$, 2-hour plasma glucose $\geq 200 \mathrm{mg} / \mathrm{dL}$ during an oral glucose tolerance test, or use of antidiabetic medications. ${ }^{16}$ Hypertension was defined as systolic blood pressure $\geq 140$ $\mathrm{mmHg}$, diastolic blood pressure $\geq 90 \mathrm{mmHg}$, or use of antihy- pertensive drugs. ${ }^{17}$ Dyslipidemia was defined as any one of the following: total cholesterol $\geq 240 \mathrm{mg} / \mathrm{dL}$, triglyceride $\geq 150$ $\mathrm{mg} / \mathrm{dL}$, low-density lipoprotein cholesterol $\geq 140 \mathrm{mg} / \mathrm{dL}$, highdensity lipoprotein cholesterol $<40 \mathrm{mg} / \mathrm{dL}$, or use of lipid-1 owering drugs. ${ }^{18}$ Income $<20 \%$ of the mean value of the total population was classified as low household income. Residential areas were divided into two groups (urban or rural), with urban areas defined as metropolitan cities with a population of $>1$ million. The smoking group included ex-smokers and current smokers, and was defined as patients who smoked at least five pack years of cigarettes in their whole lives. Alcohol consumption status was categorized as nondrinkers and alcohol drinkers, and was defined as those who drank alcohol at least once a week. Regular exercise was defined as physical activity more than three times a week for more than 20 minutes. BMI was calculated by dividing body weight by the square of the persons height, with overweight defined as BMI $>23 \mathrm{~kg} / \mathrm{m}^{2}$ and obesity as BMI $>$ $25 \mathrm{~kg} / \mathrm{m}^{2}$. Blood samples were collected after a fasting period of at

Table 1. Baseline characteristics of the study population

\begin{tabular}{lcrc}
\hline \multicolumn{1}{c}{ Variable } & Healthy population & CRC patient & $P$-value \\
\hline Sex (male) & $430,494(100)$ & $85,455(100)$ & \\
Age & & & \\
$\quad<55 \mathrm{yr}$ & $105,383(24.48)$ & $20,953(24.52)$ & $<0.0001$ \\
$\quad \geq 55 \mathrm{yr}$ & $325,111(75.52)$ & $64,502(75.48)$ & $<0.0001$ \\
Low household income ${ }^{\mathrm{a}}$ & $101,518(23.58)$ & $19,066(22.31)$ & $<0.0001$ \\
Urban residents ${ }^{\mathrm{b}}$ & $194,175(45.11)$ & $39,676(46.43)$ & $<0.0001$ \\
Diabetes mellitus & $59,992(13.94)$ & $16,692(19.53)$ & $<0.0001$ \\
Hypertension & $147,766(34.32)$ & $34,639(40.53)$ & $<0.0001$ \\
Dyslipidemia & $63,824(14.83)$ & $13,548(15.85)$ & $<0.0001$ \\
Development of prostate cancer & $4,415(1.03)$ & $2,005(2.35)$ & $<0.0001$ \\
\hline
\end{tabular}

Values are presented as number (\%). CRC, colorectal cancer. ${ }^{a}$ The low household income refers to those who are in the bottom $20 \%$ of the total population. ${ }^{b}$ Urban residents refer to people living in metropolitan areas with a population of over 1 million.

Table 2. IR and HR for development of secondary prostate cancer after adjustment for confounding factors

\begin{tabular}{|c|c|c|c|c|c|c|}
\hline \multirow{2}{*}{ Population } & \multirow{2}{*}{$\begin{array}{c}\text { Development } \\
\text { of PC }\end{array}$} & \multirow{2}{*}{ Duration $^{\mathrm{a}}$} & \multirow{2}{*}{$\mathrm{IR}^{\mathrm{b}}$} & \multicolumn{3}{|c|}{$\mathrm{HR}(95 \% \mathrm{CI})$} \\
\hline & & & & Total & Age $<55$ yr & Age $\geq 55$ yr \\
\hline \multicolumn{7}{|l|}{ Total population } \\
\hline Control group $(\mathrm{n}=430,494)$ & 4,415 & $2,506,315$ & 1.76 & 1 (ref) & 1 (ref) & 1 (ref) \\
\hline CRC patients $(\mathrm{n}=85,455)$ & 2,005 & 494,191 & 4.06 & $2.30^{c}(2.18-2.43)$ & $8.95^{c}(7.33-10.92)$ & $2.07^{c}(1.96-2.19)$ \\
\hline$P$-value & & & & $<0.0001$ & $<0.0001$ & $<0.0001$ \\
\hline \multicolumn{7}{|l|}{ Subgroup analysis } \\
\hline Control group $(\mathrm{n}=110,289)$ & 4,415 & 490,643 & 1.58 & 1 (ref) & 1 (ref) & 1 (ref) \\
\hline CRC patients $(\mathrm{n}=21,823)$ & 441 & 96,692 & 4.56 & $2.90^{\mathrm{d}}(2.58-3.27)$ & $20.74^{\mathrm{d}}(11.81-36.41)$ & $2.55^{\mathrm{d}}(2.25-2.88)$ \\
\hline$P$-value & & & & $<0.0001$ & $<0.0001$ & $<0.0001$ \\
\hline
\end{tabular}

IR, incidence rate; PC, prostate cancer; ref, reference value; CRC, colorectal cancer. ${ }^{\mathrm{a}}$ The unit of duration is person-year. ${ }^{\mathrm{b}} \mathrm{IR}$ means the number of prostate cancer patients per 1,000 people-years. 'Adjusted by age. ${ }^{\mathrm{d}}$ Adjusted for age, sex, body mass index, drink, exercise, income, diabetes mellitus, hypertension, dyslipidemia. 
least 8 hours.

\section{Statistical analysis}

Propensity score matching was used to generate the control group. Continuous variables with a normal distribution were

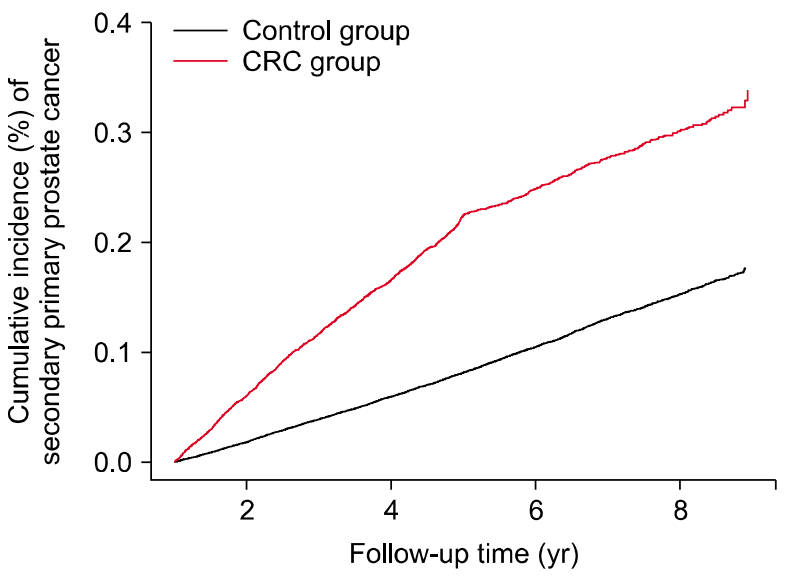

Figure 2. Cumulative incidence of prostate cancer in colorectal cancer (CRC) patients and general population. analyzed using the Student's t-test. HRs and 95\% CIs were calculated via statistical analysis using the Cox regression models after controlling for age, sex, BMI, smoking, alcohol consumption, exercise, diabetes mellitus, hypertension, dyslipidemia, and income. All statistical analyses were performed using SAS version 9.4 (SAS Institute, Cary, NC, USA) and R version 3.2.3 (The R Foundation for Statistical Computing, Vienna, Austria; http:// www. Rproject.org). A two-sided $P$-value of $<0.05$ was considered to be statistically significant.

\section{RESULTS}

\section{Demographics}

We analyzed 430,494 men without CRC and 110,289 healthy males who were registered in the NHIS database between 2007 and 2012. After excluding individuals who were diagnosed with a previous malignancy within 1 year of CRC, 85,455 male CRC patients and age-matched 430,494 control subjects were finally included in the analysis (Fig. 1). The basic demographics of this cohort are presented in Table 1. In the CRC group, the proportions of diabetes mellitus, hypertension, and dyslipidemia (all $P<$

Table 3. General characteristics of the study population (health checkup)

\begin{tabular}{|c|c|c|c|}
\hline Variable & Healthy population $(n=110,289)$ & CRC patient $(n=21,823)$ & $P$-value \\
\hline Sex (male) & $110,289(100)$ & $21,823(100)$ & \\
\hline Age & & & 0.1494 \\
\hline$<55 \mathrm{yr}$ & $27,046(24.52)$ & $5,452(24.98)$ & \\
\hline$\geq 55 \mathrm{yr}$ & $83,243(75.48)$ & $16,371(75.02)$ & \\
\hline BMI & & & 0.2922 \\
\hline$<18.5 \mathrm{~kg} / \mathrm{m}^{2}$ & $6,375(5.78)$ & $1,337(6.13)$ & \\
\hline $18.5-23 \mathrm{~kg} / \mathrm{m}^{2}$ & $46,074(41.78)$ & $9,110(41.74)$ & \\
\hline $23-25 \mathrm{~kg} / \mathrm{m}^{2}$ & $23,687(21.48)$ & $4,656(21.34)$ & \\
\hline $25-30 \mathrm{~kg} / \mathrm{m}^{2}$ & $29,064(26.35)$ & $5.689(26.07)$ & \\
\hline $25-30 \mathrm{~kg} / \mathrm{m}^{2}$ & $5,089(4.61)$ & $1,031(4.72)$ & \\
\hline Ever smoker $(y e s)^{a}$ & $39.979(36.25)$ & $7.989(36.61)$ & 0.3138 \\
\hline Drinking alcohol $>1 /$ wk (yes) & $46,298(41.98)$ & $9,277(42.51)$ & 0.1463 \\
\hline Regular exercise (yes) ${ }^{b}$ & $22,578(20.47)$ & $4,379(20.07)$ & 0.1742 \\
\hline Diabetes mellitus & $23,353(21.17)$ & $5.669(25.98)$ & $<0.0001$ \\
\hline Hypertension & 50,395 (45.69) & $11,042(50.60)$ & $<0.0001$ \\
\hline Dyslipidemia & $28,464(25.81)$ & $5,849(26.80)$ & 0.0022 \\
\hline Development of prostate cancer & $773(0.7)$ & $441(2.02)$ & $<0.0001$ \\
\hline Height $(\mathrm{cm})$ & $161.4 \pm 9.4$ & $161.5 \pm 9.4$ & 0.1185 \\
\hline Weight (kg) & $61.5 \pm 11.6$ & $61.5 \pm 11.8$ & 0.8463 \\
\hline BMI $\left(\mathrm{kg} / \mathrm{m}^{2}\right)$ & $23.5 \pm 3.6$ & $23.5 \pm 3.6$ & 0.3341 \\
\hline SBP (mmHg) & $122 \pm 15.9$ & $122.1 \pm 15.9$ & 0.8874 \\
\hline DBP (mmHg) & $75.9 \pm 10.3$ & $75.9 \pm 10.3$ & 0.9944 \\
\hline $\mathrm{TC}(\mathrm{mg} / \mathrm{dL})$ & $191.2 \pm 38.4$ & $191 \pm 38.7$ & 0.5146 \\
\hline
\end{tabular}

Values are presented as number (\%) or mean $\pm \mathrm{SD}$. The subjects of this analysis are those who have health check-up data before the diagnosis of colorectal cancer. CRC, colorectal cancer; BMI, body mass index; SBP, systolic blood pressure; DBP, diastolic blood pressure; TC, total cholesterol. ${ }^{a}$ Ever smoker is a person who has smoked at least five packs of cigarettes in their lives with the sum of ex-smoker and current smoker. ${ }^{b}$ Regular exercise refers to a person exercising at least three times a week. 
Table 4. Incidence rate of SPPC by age according to CRC

\begin{tabular}{|c|c|c|c|c|c|c|}
\hline Age (yr) & Group & Number & Development of SPPC & Duration $^{\mathrm{a}}$ & $\mathrm{IR}^{\mathrm{b}}$ & $\mathrm{HR}(95 \% \mathrm{CI})$ \\
\hline \multirow[t]{2}{*}{$<60 \mathrm{~s}$} & Control group & 163,927 & 431 & $953,860.87$ & 0.45185 & 1 (ref) \\
\hline & CRC patients & 32,603 & 502 & $188,512.34$ & 2.66296 & $5.922(5.207-6.736)$ \\
\hline \multirow[t]{2}{*}{$60 \mathrm{~s}$} & Control group & 138,339 & 1720 & $815,065.91$ & 2.11026 & $2.467(2.15-2.83)$ \\
\hline & CRC patients & 27,404 & 764 & $160,157.54$ & 4.7703 & $5.581 \quad(4.817-6.466)$ \\
\hline \multirow[t]{2}{*}{$70 \mathrm{~s}$} & Control group & 103,808 & 1950 & $598,340.56$ & 3.25901 & $2.439(2.029-2.933)$ \\
\hline & CRC patients & 20,586 & 642 & $117,938.96$ & 5.44349 & $4.081 \quad(3.358-4.96)$ \\
\hline \multirow[t]{2}{*}{$80 \mathrm{~s}$} & Control group & 24,420 & 314 & $139,048.39$ & 2.25821 & $1.044(0.8-1.363)$ \\
\hline & CRC patients & 4,862 & 97 & $27,583.04$ & 3.51665 & $1.625(1.188-2.223)$ \\
\hline
\end{tabular}

These results are obtained by health checkup data. SPPC, secondary primary prostate cancer; CRC, colorectal cancer; IR, incidence rate; ref, reference value. ${ }^{\mathrm{a}}$ The unit of duration is person-year. ${ }^{\mathrm{b}} \mathrm{IR}$ means the number of prostate cancer patients per 1,000 people-years.

$0.0001)$ were significantly higher than those in the control group. Subjects whose yearly income was in the bottom $20 \%$ among the total population, were significantly less common in the CRC group than general population. The proportion of residents in urban areas was significantly higher in the CRC group than general population.

\section{Risk for development of secondary primary prostate cancers in colorectal cancer patients}

During the median follow-up period of 5.78 years, 2,005 patients (2.30\%) developed SPPC in the CRC group, while 4,415 subjects with prostate cancer (1.03\%) were confirmed in the control group. The Cox regression model was used to predict the potential risk factors for SPPC. The incidence rate (IR) of SPPC among CRC patients was 4.06/1,000 person-years and HR was 2.30 (95\% CI, 2.18-2.43); HR was higher in subjects aged $<55$ years than those aged $\geq 55$ years (HR, 8.95; 95\% CI, 7.33-10.92) (Table 2).

The cumulative incidence of prostate cancer in each group over time is presented in Figure 2. The cumulative incidence of SPPC among CRC patients was $0.2 \%$ at 5 years after diagnosis. The cumulative incidence of SPPC in CRC patients was continuously higher than that in the general population, representing $0.05 \%$ at 5 years after the diagnosis of CRC (IR 4.06 vs. 1.76/1,000 person-years, respectively).

\section{Subgroup analysis}

Among CRC patients who underwent health checkups within 1 year before CRC diagnosis, 21,823 patients were matched to 110,289 subjects in the control group using the health checkup data according to age at a 1:5 ratio. The basic demographics are presented in Table 3. Individuals with diabetes mellitus, hypertension, and dyslipidemia (all $P<0.0001$ ) were significantly more common in the CRC group than in the control group. Current smoking, alcohol consumption, and BMI were not significantly different between both groups. We identified IR and HR of prostate cancer after adjusting for confounders. Multivariate analysis demonstrated that CRC patients remained significantly independent predictors of SPPC development (IR 4.56/ 1,000 person-years, HR 2.90; 95\% CI, 2.58-3.27) (Table 2). In this subgroup analysis, younger CRC patients, particularly those diagnosed at $<55$ years of age were significantly associated with a higher risk of SPPC (HR, 20.74; 95\% CI, 11.81-36.41). Table 4 demonstrates that the association between development of SPPC and $C R C$ is higher in younger patients who are diagnosed with CRC.

\section{DISCUSSION}

In the present nationwide population-based cohort study, we demonstrated that the incidence of SPPC is higher in CRC patients than in individuals without previous malignancies. This risk was particularly higher in men aged $<55$ years than in others.

There are several reports on SPMs among CRC survivors; however, only a few studies exist on the development of SPPC in CRC patients. Evans et al. ${ }^{2}$ demonstrated that CRC patients aged $<60$ years are at risk of SPMs in other sites. Phipps et al. ${ }^{19}$ reported a slightly increased risk of secondary non-CRC using the Surveillance, Epidemiology, and End Results (SEER) registries with a standardized incidence ratio (SIR) of 1.24. Ahmed et al. ${ }^{20}$ studied the excess risk of subsequent primary cancers among CRC survivors. They used SEER and reported the significantly elevated SIR of 26.48 in only black males. Moot et al. ${ }^{21}$ investigated the relationship between CRC and SPPC. They showed that men who develop CRC are at an increased risk of SPPC (SIR at follow-up period: $<1$ year, 1.7; 1-5 years, 1.3; 5-10 years, 1.4), with the highest risk in men aged $<65$ years. Furthermore, Lee et $\mathrm{al}^{4}{ }^{4}$ reported that the risk of SPPC was 1.2 times higher in CRC patients than general population. 
The mechanism of the increased risk for SPPC in patients has not yet been proved. Several etiologies, including existing background, lifestyle, comorbidities, and environmental components are related to the development of SPPC. For example, hereditary nonpolyposis CRC patients tend to develop other extracolonic malignancies more than the general population. ${ }^{22}$ In addition, there may be unknown shared etiological factors that are involved in the link between the CRC and SPPC. ${ }^{23-25}$ SPPC development may also be related to daily lifestyle factors, such as saturated fat intake, which has been implicated in both CRC and prostate cancer. ${ }^{26-28}$ In addition, the treatment of CRC can cause the development of SPMs. For example, chemotherapy and radiation are related to SPMs; however, the exact mechanism is not yet proved. ${ }^{29.30}$

Another explanation is that the increased incidence of SPPC may result from a screening detection bias, which has been suggested. ${ }^{31}$ Men previously diagnosed with CRC can be followedup more closely than those without any previous malignancies, including frequent digital rectal examinations. Given that CRC survivors were twice as risky as the general population without previous malignancies, and that this risk was higher in young patients, the importance of screening or surveillance is raised. However, there is considerable doubt whether physicians will be able to identify a curable lesion localized to the prostate gland. Although it is uncertain whether blood tests using prostatespecific antigens are effective for screening CRC survivors, ${ }^{32-34}$ our results suggest that further studies are required to determine the link between CRC and SPPC, as well as strategies for prevention.

The present study had several limitations. First, we did not classify CRC patients according to treatment based on the stage of the disease, making it impossible to assess the correlation between disease severity, treatment modality, and incidence of SPPC. Second, the development of prostate cancer is time demanding; therefore, a longer follow-up period would have yielded a remarkable result. Finally, in addition to the BMI, smoking, alcohol consumption, exercise, hypertension, and diabetes mellitus, there are many other related factors between CRC and SPPC, such as lifestyle, eating habits, family history etc. In this point of view, bias exists and it is worth mentioning as a limitation. There are some advantages of this study. This is a population-based nationwide study using the NHIS database. This database records the claims information of approximately $97 \%$ of all Koreans. Therefore, it represents almost the entire Korean population. Because all patients registered for cancer require a confirmation by biopsy, the record of the diagnosis of cancer is reliable. In addition, we analyzed health checkup data for the first time. Using the health checkup claim data, we adjusted for potential confounding factors (BMI, diabetes mellitus, dyslipidemia, smoking, and alcohol consumption) that could affect the progression to prostate cancer.

In conclusion, this nationwide population-based study suggests that CRC patients are at an increased risk of SPPC, especially young patients aged $<55$ years. Early detection of prostate cancer, which has an elevated likelihood of occurring in CRC patients, is important. Therefore, as next steps, we suggest implementation of proper screening techniques for prostate cancer and investigating the possible shared etiologies and mechanisms of carcinogenesis.

\section{ACKNOWLEDGMENTS}

This work was supported by Korea Institute of Planning and Evaluation for Technology in Food, Agriculture, Forestry and Fisheries (IPET) through (High Value-added Food Technology Development Program), funded by Ministry of Agriculture, Food and Rural Affairs (MAFRA) (No. 116017032HD030).

\section{CONFLICTS OF INTEREST}

No potential conflicts of interest were disclosed.

\section{REFERENCES}

1. Fitzmaurice C, Allen C, Barber RM, Barregard L, Bhutta ZA, Brenner H, et al.; Global Burden of Disease Cancer Collaboration. Global, regional, and national cancer incidence, mortality, years of life lost, years lived with disability, and disability-adjusted life-years for 32 cancer groups, 1990 to 2015: a systematic analysis for the global burden of disease study. JAMA Oncol 2017;3:524-48.

2. Evans HS, Møller H, Robinson D, Lewis CM, Bell CM, Hodgson SV. The risk of subsequent primary cancers after colorectal cancer in southeast England. Gut 2002;50:647-52.

3. Mariotto AB, Rowland JH, Ries LA, Scoppa S, Feuer EJ. Multiple cancer prevalence: a growing challenge in long-term survivorship. Cancer Epidemiol Biomarkers Prev 2007; 16:566-71.

4. Lee YT, Liu CJ, Hu YW, Teng CJ, Tzeng CH, Yeh CM, et al. Incidence of second primary malignancies following colorectal cancer: a distinct pattern of occurrence between colon and rectal cancers and association of co-morbidity with second primary malignancies in a population-based cohort of 98,876 patients in Taiwan. Medicine (Baltimore) 2015;94:e1079.

5. Litwin MS, Tan HJ. The diagnosis and treatment of prostate cancer: a review. JAMA 2017;317:2532-42.

6. Esposito K, Chiodini P, Colao A, Lenzi A, Giugliano D. Metabolic syndrome and risk of cancer: a systematic review and metaanalysis. Diabetes Care 2012;35:2402-11.

7. Esposito K, Chiodini P, Capuano A, Bellastella G, Maiorino MI, 
Parretta E, et al. Effect of metabolic syndrome and its components on prostate cancer risk: meta-analysis. J Endocrinol Invest 2013;36:132-9.

8. Teppo L, Pukkala E, Saxén E. Multiple cancer: an epidemiologic exercise in Finland. J Natl Cancer Inst 1985;75:207-17.

9. Multiple primary cancers in Connecticut and Denmark. Natl Cancer Inst Monogr 1985;68:1-437.

10. Enblad P, Adami HO, Glimelius B, Krusemo U, Påhlman L. The risk of subsequent primary malignant diseases after cancers of the colon and rectum. A nationwide cohort study. Cancer 1990; 65:2091-100.

11. McCredie M, Macfarlane GJ, Bell J, Coates M. Second primary cancers after cancers of the colon and rectum in New South Wales, Australia, 1972-1991. Cancer Epidemiol Biomarkers Prev 1997;6:155-60.

12. Cluze C, Delafosse P, Seigneurin A, Colonna M. Incidence of second cancer within 5 years of diagnosis of a breast, prostate or colorectal cancer: a population-based study. Eur J Cancer Prev 2009; 18:343-8.

13. Hamer M, Stamatakis E. Metabolically healthy obesity and risk of all-cause and cardiovascular disease mortality. J Clin Endocrinol Metab 2012;97:2482-8.

14. Ortega FB, Lee DC, Katzmarzyk PT, Ruiz JR, Sui X, Church TS, et al. The intriguing metabolically healthy but obese phenotype: cardiovascular prognosis and role of fitness. Eur Heart J 2013;34:389-97

15. Song SO, Jung CH, Song YD, Park CY, Kwon HS, Cha BS, et al. Background and data configuration process of a nationwide population-based study using the Korean National Health Insurance System. Diabetes Metab J 2014:38:395-403.

16. American Diabetes Association. Diagnosis and classification of diabetes mellitus. Diabetes Care 2010;33:S62-9.

17. James PA, Oparil S, Carter BL, Cushman WC, DennisonHimmelfarb C, Handler J, et al. 2014 evidence-based guideline for the management of high blood pressure in adults: report from the panel members appointed to the Eighth Joint National Committee (JNC 8). JAMA 2014:311:507-20.

18. Teramoto T, Sasaki J, Ueshima H, Egusa G, Kinoshita M, Shimamoto K, et al. Diagnostic criteria for dyslipidemia. Executive summary of Japan Atherosclerosis Society (JAS) guideline for diagnosis and prevention of atherosclerotic cardiovascular diseases for Japanese. J Atheroscler Thromb 2007; 14:155-8.

19. Phipps AI, Chan AT, Ogino S. Anatomic subsite of primary colorectal cancer and subsequent risk and distribution of second cancers. Cancer 2013;119:3140-7.

20. Ahmed F, Goodman MT, Kosary C, Ruiz B, Wu XC, Chen VW, et al. Excess risk of subsequent primary cancers among colorectal carcinoma survivors, 1975-2001. Cancer 2006;107(5 Suppl):1162-71.

21. Moot AR, Polglase A, Giles GG, Garson OM, Thursfield V, Gunter D. Men with colorectal cancer are predisposed to prostate cancer. ANZ J Surg 2003:73:289-93.

22. Zauber AG, Winawer SJ, O'Brien MJ, Lansdorp-Vogelaar I, van Ballegooijen M, Hankey BF, et al. Colonoscopic polypectomy and long-term prevention of colorectal-cancer deaths. N Engl J Med 2012;366:687-96.

23. Bookstein R, Bova GS, MacGrogan D, Levy A, Isaacs WB. Tumour-suppressor genes in prostatic oncogenesis: a positional approach. Br J Urol 1997;79 Suppl 1:28-36.

24. MacGrogan D, Pegram M, Slamon D, Bookstein R. Comparative mutational analysis of DPC4 (Smad4) in prostatic and colorectal carcinomas. Oncogene 1997;15:1111-4.

25. Bookstein R. Tumor suppressor genes in prostatic oncogenesis. J Cell Biochem Suppl 1994;19:217-23.

26. Whittemore AS, Kolonel LN, Wu AH, John EM, Gallagher RP, Howe GR, et al. Prostate cancer in relation to diet, physical activity, and body size in blacks, whites, and Asians in the United States and Canada. J Natl Cancer Inst 1995;87:652-61.

27. Kolonel LN, Nomura AM, Cooney RV. Dietary fat and prostate cancer: current status. J Natl Cancer Inst 1999:91:414-28.

28. Glade MJ. Food, nutrition, and the prevention of cancer: a global perspective. American Institute for Cancer Research/World Cancer Research Fund, American Institute for Cancer Research, 1997. Nutrition 1999;15:523-6.

29. Reulen RC, Frobisher C, Winter DL, Kelly J, Lancashire ER, Stiller CA, et al. Long-term risks of subsequent primary neoplasms among survivors of childhood cancer. JAMA 2011;305:2311-9.

30. Berrington de Gonzalez A, Curtis RE, Kry SF, Gilbert E, Lamart S, Berg $\mathrm{CD}$, et al. Proportion of second cancers attributable to radiotherapy treatment in adults: a cohort study in the US SEER cancer registries. Lancet Oncol 2011;12:353-60.

31. Hoar SK, Wilson J, Blot WJ, McLaughlin JK, Winn DM, Kantor AF. Second cancer following cancer of the digestive system in Connecticut, 1935-82. Natl Cancer Inst Monogr 1985:68:49-82.

32. Meyer F, Moore L, Bairati I, Fradet Y. Downward trend in prostate cancer mortality in Quebec and Canada. J Urol 1999;161:1189-91.

33. Burack RC, Wood DP Jr. Screening for prostate cancer. The challenge of promoting informed decision making in the absence of definitive evidence of effectiveness. Med Clin North Am 1999;83:1423-42.

34. Oliver SE, May MT, Gunnell D. International trends in prostate-cancer mortality in the "PSA ERA". Int J Cancer 2001;92:893-8. 